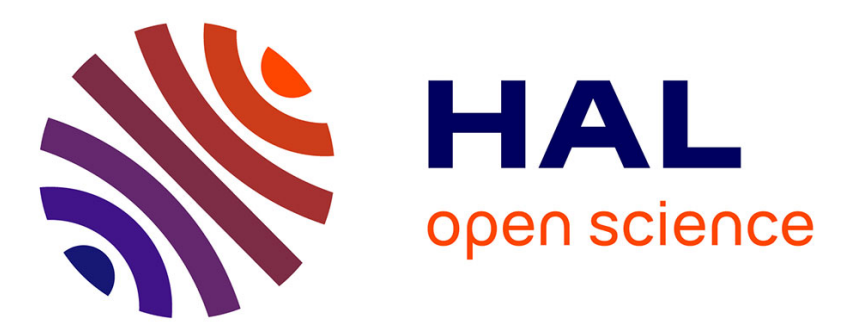

\title{
Size and Origins of Long-Range Orientational Water Correlations in Dilute Aqueous Salt Solutions
}

\author{
Eva Pluhařová, Damien Laage, Pavel Jungwirth
}

\section{To cite this version:}

Eva Pluhařová, Damien Laage, Pavel Jungwirth. Size and Origins of Long-Range Orientational Water Correlations in Dilute Aqueous Salt Solutions. Journal of Physical Chemistry Letters, 2017, 8, pp.2031 - 2035. 10.1021/acs.jpclett.7b00727 . hal-01516870

\section{HAL Id: hal-01516870 https://hal.sorbonne-universite.fr/hal-01516870}

Submitted on 2 May 2017

HAL is a multi-disciplinary open access archive for the deposit and dissemination of scientific research documents, whether they are published or not. The documents may come from teaching and research institutions in France or abroad, or from public or private research centers.
L'archive ouverte pluridisciplinaire HAL, est destinée au dépôt et à la diffusion de documents scientifiques de niveau recherche, publiés ou non, émanant des établissements d'enseignement et de recherche français ou étrangers, des laboratoires publics ou privés. 


\title{
On the Size and Origins of Long-Range
}

\section{Orientational Water Correlations in Dilute Aqueous}

\section{Salt Solutions}

\author{
Eva Pluhařová ${ }^{1,2^{*}}$, Damien Laage ${ }^{1 *}$, and Pavel Jungwirth ${ }^{3 *}$ \\ ${ }^{1}$ École Normale Supérieure - PSL Research University, Département de Chimie, Sorbonne \\ Universités - UPMC Univ Paris 06, CNRS UMR 8640 PASTEUR, 24 rue Lhomond, 75005 \\ Paris, France
}

${ }^{2}$ J. Heyrovský Institute of Physical Chemistry, Czech Academy of Sciences, v.v.i., Dolejškova 3, Prague, 18223, Czech Republic

${ }^{3}$ Institute of Organic Chemistry and Biochemistry, Czech Academy of Sciences, Flemingovo nám. 2, Prague, 16610, Czech Republic

\author{
AUTHOR INFORMATION \\ *Corresponding authors: eva.pluharova@jh-inst.cas.cz, damien.laage@ens.fr, and \\ pavel.jungwirth@uochb.cas.cz
}




\begin{abstract}
Long-range ordering of water around solutes has been repeatedly invoked as the key to its biological function. Recently, it has been shown that in a $8 \mathrm{mM}$ aqueous $\mathrm{NaCl}$ solution the orientational correlation between water molecules extends beyond 8 nanometers. This was interpreted as arising from ion-induced long-range effects on the water collective hydrogen-bond interactions. Each ion was suggested to affect $>10000$ water molecules, leading to a picture involving nanoscopic "ordered domains". Using molecular dynamics simulations, we show that the very small long-range tail in the correlation function is caused primarily by pairs of water molecules belonging to different ions' hydration shells and can be made to practically disappear by rearranging the ionic positions. This means that the ion-induced water orientational ordering in millimolar salt solutions cannot be separated from ion-ion interaction effects, for which the Debye-Hückel screening length shrinks to less than a nanometer at physiological ionic strengths.
\end{abstract}

\title{
TOC GRAPHIC
}

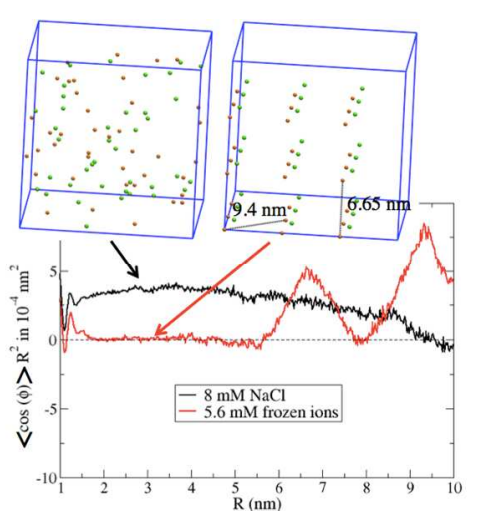


The Debye-Hückel screening length $\lambda_{\mathrm{D}}$ determines the range of mutual interactions of charged particles. ${ }^{1-3}$ More precisely, for salt ions dissolved in water the electric field decreases exponentially by a factor of $1 / \mathrm{e}$ at a distance of $\lambda_{\mathrm{D}}=0.304 I^{1 / 2} \mathrm{~nm}$, where $I$ is the ionic strength of the solution. ${ }^{1-2}$ At physiological concentrations of $\sim 150 \mathrm{mM}$ (which is, strictly speaking, beyond the quantitative validity of the Debye-Hückel theory) the screening length is about 0.8 $\mathrm{nm}$, while in a more dilute $\sim 1 \mathrm{mM}$ solutions it increases to almost $10 \mathrm{~nm}$. This sensitive dependence of the electrostatic screening of salt ions on their concentration in water is one of the cornerstones of theories of electrolytes. ${ }^{3}$

Recently, a combined experimental and computational study suggested that in dilute aqueous salt solution of about $10 \mu \mathrm{M}$ to a few $\mathrm{mM}$, long-range water-water correlations induced by the ions can be observed. ${ }^{4}$ Following the definition implicitly employed in Ref. 4 , here we will use the term "long range" to designate ion-induced structural effects that extend beyond the first few hydration layers, i.e. typically beyond $1 \mathrm{~nm}$. Simulations of a $8 \mathrm{mM} \mathrm{NaCl}$ solution showed that a small angular correlation between pairs of water molecules persists up to distances of at least $8 \mathrm{~nm}$ (limited by the employed simulation unit cell size). The interpretation provided in Ref. 4, where the simulations were performed primarily to support the accompanying experimental observations, has been that each ion in dilute salt solutions induces nanoscopic “ordered domains" containing $10^{4}-10^{6}$ surrounding water molecules. This claim may however appear to be at odds with the very short distance at which water molecules become mutually uncorrelated in the aqueous bulk. Ion-ion interaction energies become smaller than the thermal energy for separations greater than the Bjerrum length, which in room temperature water amounts only to $0.7 \mathrm{~nm} .^{5}$ Since water-ion and water-water correlations decay more rapidly than 
ion-ion interactions with increasing separation, in pure water orientational correlations become effectively negligible beyond $1 \mathrm{~nm} .{ }^{4}$ In the rest of this Letter we elaborate on this issue by means of molecular simulations. We specifically focus on the ion-induced long-range structural correlation between water molecules that was inferred from simulations in Ref. 4, while we do not address here the experimental results of Ref. 4 . We provide a careful assessment of the size of the long-range water-water orientational correlations together with a refined interpretation thereof including the effects of ion-ion correlations within the extent of the Debye-Hückel screening length.

As a first step, we reproduced the simulation results from Ref. 4 concerning a $8 \mathrm{mM}$ aqueous solution of $\mathrm{NaCl}$. Figure 1 shows the resulting distance-weighted water-water orientational correlation function $\langle\cos \theta\rangle R^{2}$ (black line), where $\theta$ is the angle between the dipoles and $R$ the distance between their respective oxygen atoms. Our results confirm that the previously reported long-range tail of the correlation function extending to distances R of almost $10 \mathrm{~nm}$ is real, albeit of a very small magnitude. It follows from basic electrostatics that orientational correlations of dipolar solvent molecules around an ion decay as $\mathrm{R}^{-2}$. Unlike the strong short-range (sub-nanometer) water-water orientational correlations, the long-range tail is thus only visible when distance weighted, i.e. multiplied by $\mathrm{R}^{2}$, as already noted in Ref. 4. Even then, the values at the tail of the correlation function are less than $5 \times 10^{-4} \mathrm{~nm}^{2}$. This value should be compared to the standard deviation of $\cos (\theta)$ multiplied by $\mathrm{R}^{2}$, which becomes many orders of magnitude larger than the distance-weighted water-water orientational correlations for distances beyond 1-2 nanometers (see Figure 2). Assuming for simplicity that this $5 \times 10^{-4} \mathrm{~nm}^{2}$ angular correlation at $4 \mathrm{~nm}$ arises from a harmonic potential $1 / 2 \mathrm{k}[\cos (\theta)-1]^{2}$, it is straightforward to show that the energy difference $\mathrm{k}$ between the parallel and antiparallel water dipole orientations 
is less than $0.0001 \mathrm{k}_{\mathrm{B}} \mathrm{T}$, which simply cannot lead to nanoscopic "ordered domains"4 in water at ambient conditions.

What is the origin of the small long-range tail of the water-water orientational correlations in dilute salt solutions? To address this issue, we first consider the case of a single ion. Depending on the ion's charge, the preferred orientation of a dipole moment of a water molecule is (anti-) parallel to the ion-water radial direction; the point charge - water dipole interaction decays in $1 / \mathrm{R}^{2}$, and the $\mathrm{R}^{2}$ distance weighting would lead to a constant distanceweighted correlation, independent of R (Fig. 2). Beyond about $1 \mathrm{~nm}$, the ion-water interaction is smaller than the thermal energy, but once the thermal fluctuations are averaged out, a small residual angular correlation persists, even though these water molecules do not form ordered domains. We now analyze dilute salt solutions and investigate which water molecules contribute to the angular correlation. We first stress that while pairs of water molecules in the vicinity of the same ion exhibit the largest correlation, the $\mathrm{R}^{2}$ distance weighting puts the emphasis on pairs of water molecules which are much more weakly correlated but whose population is larger. The inset of Figure 2 clearly demonstrates that water molecules which are found around the same ion (i.e., for which the nearest ion is the same) have a vanishing contribution beyond $2 \mathrm{~nm}$. In other words, the long-range tail originates primarily from orientational correlations between pairs of water molecules whose nearest ions are different, and which thus belong to different ion solvation shells (our analysis further shows that the largest contribution to the long-range correlation is found to come from pairs of water molecules both within 1 to $3 \mathrm{~nm}$ from their closest ions). This immediately brings the idea to try to influence the behavior of this tail by performing additional simulations at comparable salt concentrations, in which the positions of ions are initially arranged in various patterns and then kept fixed. One such ion arrangement is 
presented in Figure 1, which also shows the resulting water-water orientational correlations function (red line). While in these dilute solutions with average nearest neighbor ion-ion distances of several nanometers the short-range (sub-nanometer) correlations were insensitive to the particular ionic arrangement, the long-range correlations were not. This is demonstrated in Figure 1, which shows that for the presented ionic arrangement the long-range tail of the correlation function practically vanishes up to about $5 \mathrm{~nm}$. Moreover, the peaks at 6.65 and 9.4 $\mathrm{nm}$ match perfectly the chosen smallest and second smallest distances between like charge ions.

The above observations point directly to the fact that the long-range tail of the waterwater orientational correlation function is strongly influenced by ion-ion correlations. In other words, the ions themselves are correlated and, therefore, also their hydration shells, are orientationally correlated at the Debye-Hückel screening length, which extends in these dilute solutions to 5-10 nanometers. This is also why merely by rearranging the ions the long-range water-water orientational correlations can be completely changed (and even zeroed out for $2<\mathrm{R}$ $<5 \mathrm{~nm}$ ), see Figure 1. 


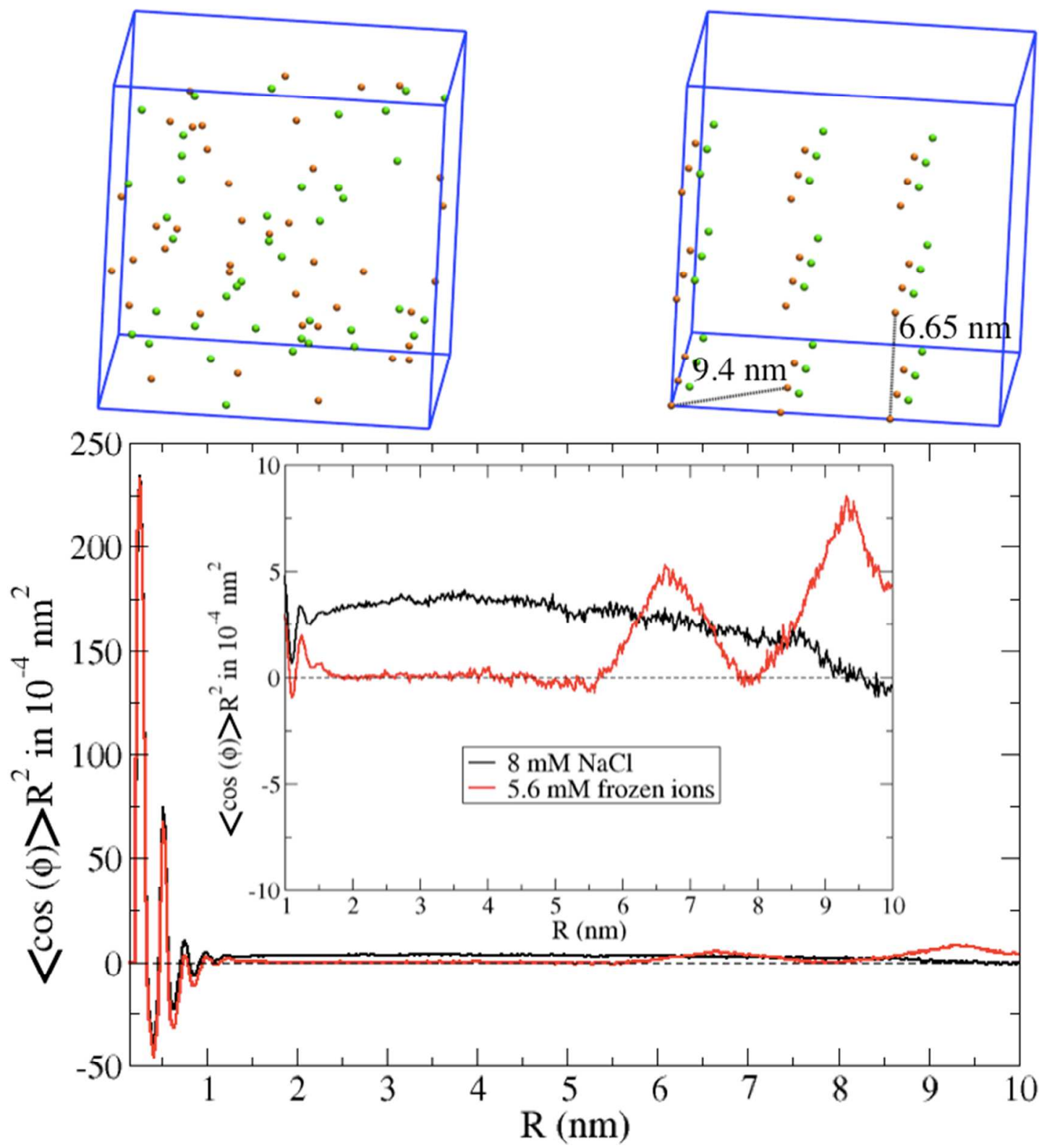

Figure 1. Top: Snapshots of ionic arrangements in an unconstrained $8 \mathrm{mM} \mathrm{NaCl}$ solution (left) and in a $5.6 \mathrm{mM} \mathrm{NaCl}$ solution with a fixed regular arrangement of ions (right). Bottom: The corresponding distance-weighted water-water orientational correlation functions (black and red lines). The inset zooms in the correlation for distances larger than $1 \mathrm{~nm}$. 


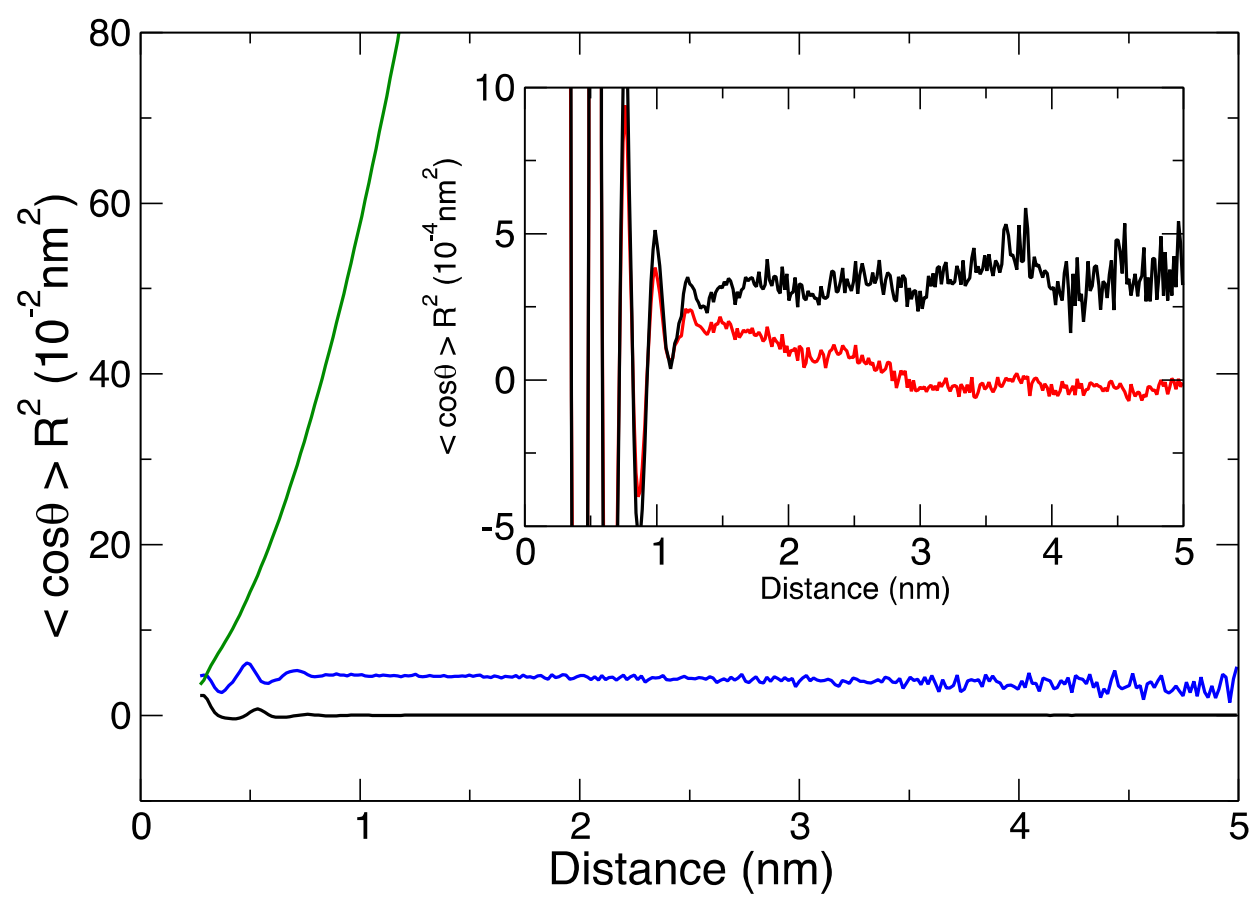

Figure 2. Comparison between the distance-weighted long-range water-water orientational correlations $\langle\cos \theta\rangle R^{2}$ in an unconstrained $8 \mathrm{mM} \mathrm{NaCl}$ solution (black) and the corresponding distance-weighted standard deviation $\sqrt{\left\langle[\cos \theta]^{2}\right\rangle-\langle\cos \theta\rangle^{2}} R^{2}$ in green (which is very close to the isotropic limit of $R^{2} \sqrt{1 / 3}$ ). The blue curve depicts the angular correlation between the water dipole and the vector between the water oxygen and the nearest cation as a function of the ionwater distance, demonstrating how small this long-range orientational correlation, and even more so the long-range water-water orientational correlation, is compared to the standard deviation of the cosine of the corresponding angle. The inset shows again the distance-weighted long-range water-water orientational correlations (black) together with the contribution from water molecules around the same ion (red), which vanishes beyond $2 \mathrm{~nm}$. 
From the technical point of view, it is important to note that the exact shape of the longrange tail of the water-water orientational correlation function can depend sensitively on the boundary conditions chosen for the simulations. Indeed, Figure 3 demonstrates that in pure water the often utilized tin foil Ewald boundary condition, ${ }^{4,6-7}$ corresponding to a dielectric constant $\varepsilon_{\mathrm{r}}$ ' $=\infty$, leads to a spurious increase of the distance-weighted water-water orientational correlations at large distances. This artifact decreases with increasing system size, nevertheless, it is still clearly present even for the largest employed $20 \mathrm{~nm}$ simulation cell. In fact, the size of this artifact in neat water is comparable to the effect of millimolar salt on the tail of the correlation function discussed above (Figure 1). For example, at distances of $5 \mathrm{~nm}$ or more, we found that the magnitude of the observed ion-induced correlation changes by more than a factor of 2 with the choice of boundary conditions, despite the large $20 \mathrm{~nm}$ dimension of the simulation box. The proper way to deal with this issue ${ }^{8}$ is to set the value of the dielectric constant at the unit cell boundary to that corresponding to the simulated water model, i.e., about $60 .{ }^{9} \mathrm{We}$ note that the artifacts due to the tin foil boundary condition tend to disappear as salt is added to the solution changing it from an insulator to a conductor provided a sufficiently large unit cell is employed. ${ }^{4}$ 


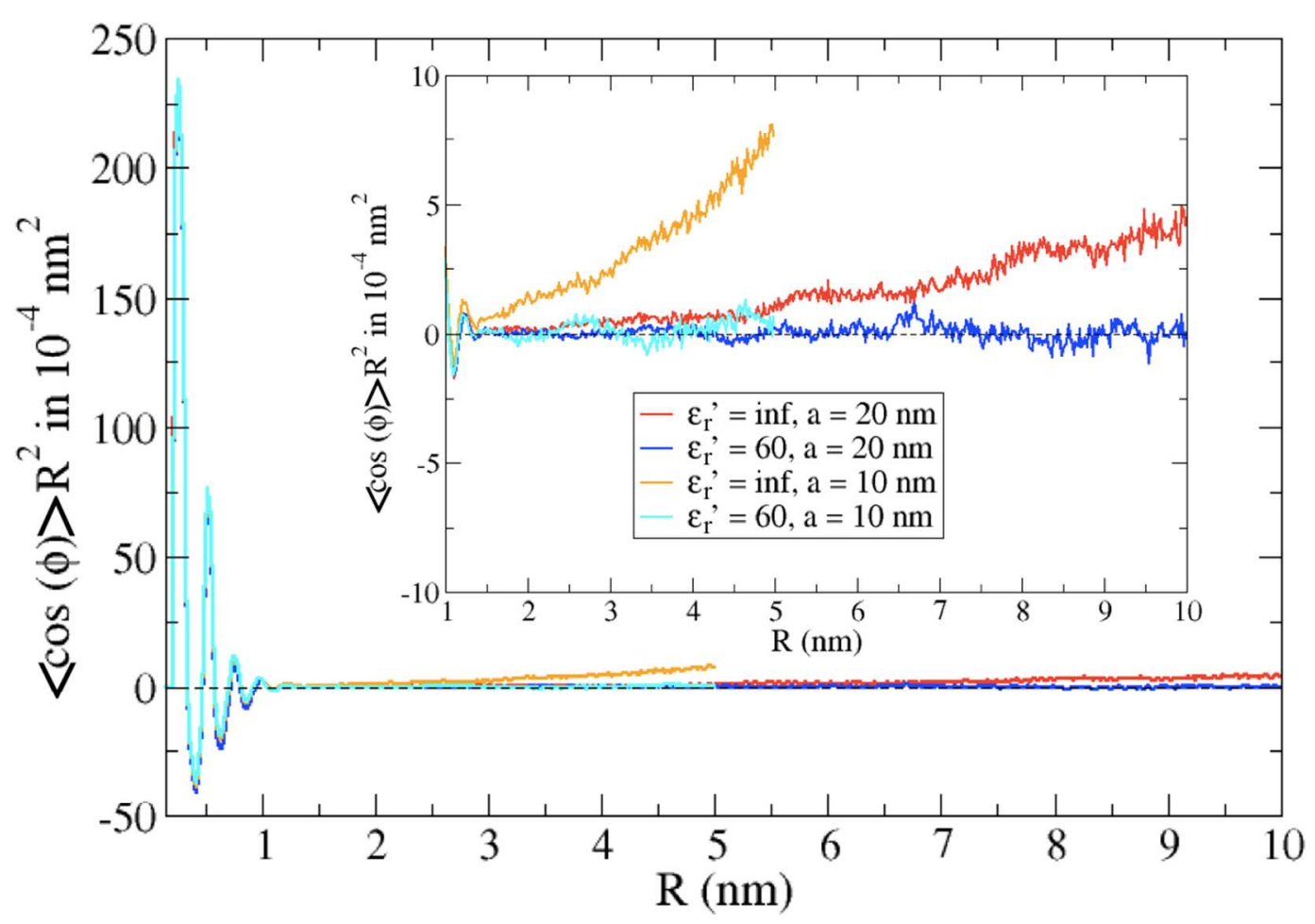

Figure 3. Distance-weighted water-water orientational correlation functions in neat water. Comparison of two types of Ewald boundary conditions - a tin foil $\varepsilon_{\mathrm{r}}{ }^{\prime}=\infty$ (red) vs. $\varepsilon_{\mathrm{r}}{ }^{\prime}=60$ (blue) for two sizes of the unit cell of approximately 10 and $20 \mathrm{~nm}$. The inset zooms in the correlation functions for distances larger than $1 \mathrm{~nm}$.

Our conclusion that the small long-range tail in the water-water orientational correlation function is strongly influenced by ion arrangements is further supported by its concentration dependence behavior. Figure 4 presents the results obtained from simulations with a unit cell size of $10 \mathrm{~nm}$ for four concentrations from $17 \mathrm{mM}$ to $1 \mathrm{M}$, compared to neat water. While for shorter distances the correlation function increases with the amount of dissolved salt, at larger distances it decays faster at higher concentration. For physiological concentrations the distance-weighted orientational correlation function becomes negligible beyond $\sim 2 \mathrm{~nm}$, while at $1 \mathrm{M}$ the 
electrostatic screening is such that the correlation function vanishes beyond $1 \mathrm{~nm}$ similarly as in neat water.

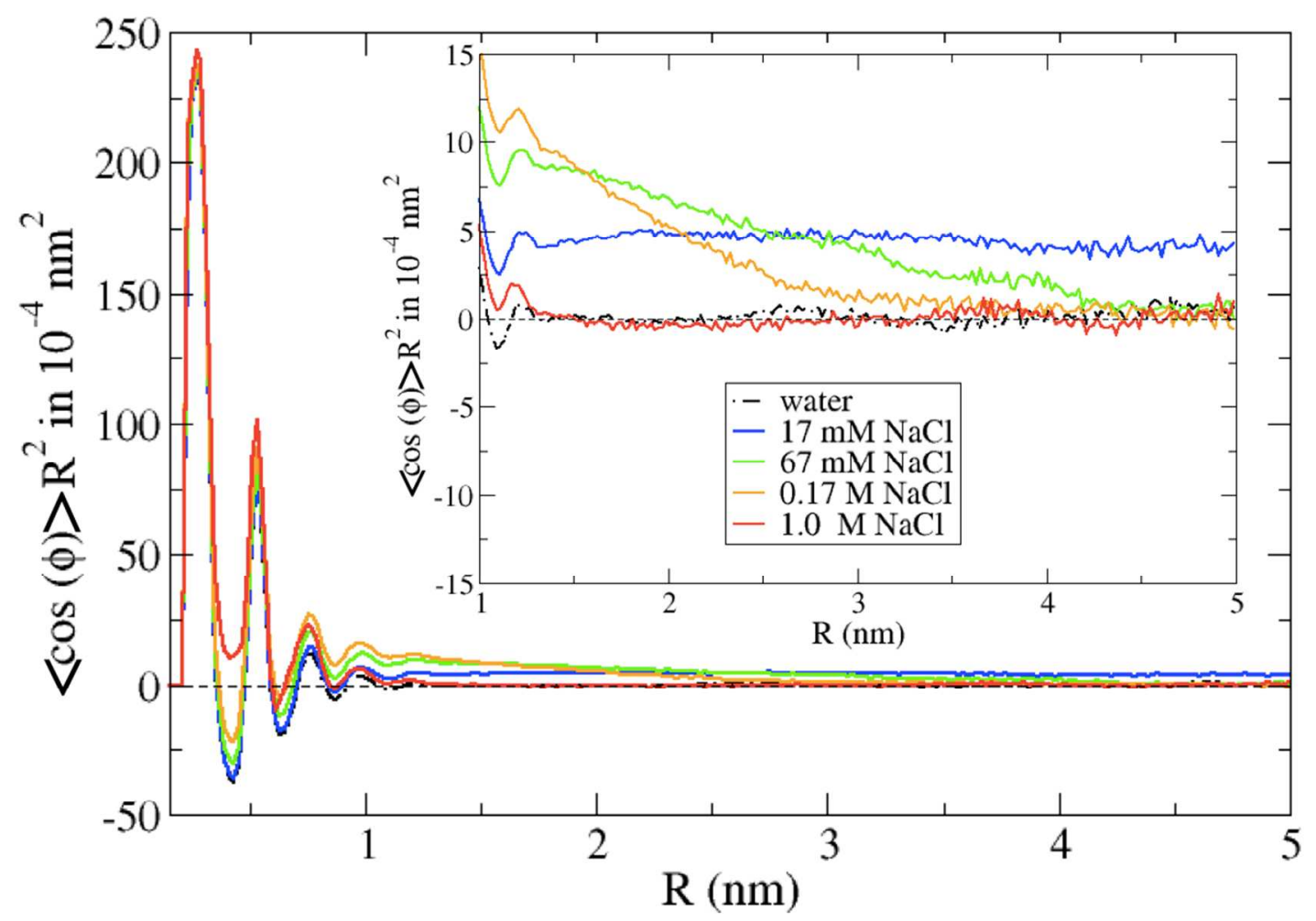

Figure 4. Concentration dependence of distance weighted water-water orientational correlation in a $\sim 10$ nm simulation cell, comparison of neat water (black dashed-dotted line) and solutions with different $\mathrm{NaCl}$ concentration (color solid lines). The inset zooms in the correlation for distances larger than $1 \mathrm{~nm}$.

The importance of our present findings goes beyond an academic discussion about the proper interpretation of the small long-range tail of the water-water orientational correlation function in dilute salt solutions. The relevance of long-range effects in aqueous solutions have been widely debated, and it has been claimed for example that long-range "allosteric" effects in 
water could be a possible key to its biological function. ${ }^{10-11}$ While Ref. 4 does not join such claims, its description involving tens of thousands or more water molecules organized around an ion and forming nanoscopic "ordered domains", may be viewed as overreaching. Our present study shows that the small ion-induced structural correlation between water molecules can be explained within a more sober picture which does not support the notion of long-range effects in the role of water in biological environments. The influence of ions is only important for water molecules in their vicinity and correlations between water molecules are of a local character. The small long-range tail in the correlation function is due to correlations between water molecules belonging to solvent shells of different ions and by simply rearranging the ionic positions it can be effectively attenuated. This tail thus strongly depends on ion-ion correlations the extent of which is given by the Debye-Hückel screening length. This length is of the order of 10 nanometers in millimolar salt solutions, but shortens to less than $1 \mathrm{~nm}$ at a physiological ionic strength. Our findings reinforce the notion of water as a solvent that responds to solute molecules locally, leaving potential long-range allosteric effects to the solutes themselves.

\section{COMPUTATIONAL METHODS}

First, we performed molecular dynamics simulations for the same diluted aqueous salt solution as in Ref. 4. Namely, the simulated system consisted of 264363 TIP4P/2005 water molecules ${ }^{12}$ with $40 \mathrm{Na}^{+}$and $40 \mathrm{Cl}^{-}$ions described by the CHARMM36 force field. ${ }^{13}$ We have shown in our previous study that such a non-polarizable force field performs satisfactorily for this system, where effects of electronic polarization are modest. ${ }^{14}$ The geometry of water molecules were kept rigid by the SETTLE algorithm. The equations of motion were integrated using the leap-frog algorithm with a time step of 2 fs using the Gromacs 4.6 .5 software. ${ }^{15}$ Threedimensional periodic boundary conditions were applied. Short range electrostatic and van der 
Waals interactions were truncated at $0.9 \mathrm{~nm}$, with the long range electrostatic interactions being accounted for via the smooth particle mesh Ewald method with a grid spacing of $0.12 \mathrm{~nm}$ employing tin foil Ewald boundary conditions. ${ }^{16}$ We collected ten 5 ns trajectories (50 ns in total) in an NVT ensemble at the temperature of $298 \mathrm{~K}$ maintained by the canonical velocity rescaling thermostat ${ }^{17}$ with a coupling constant of $1 \mathrm{ps}$. The total simulation length was sufficient to converge the water-water orientational correlation function, which was sampled every 2 ps.

Initial configurations were taken from a 2 ns equilibration at a constant pressure of $1 \mathrm{bar}$ using the Berendsen barostat ${ }^{18}$ with a coupling constant of 0.5 ps and at constant temperature of $298 \mathrm{~K}$ using the canonical velocity rescaling thermostat ${ }^{17}$ with a coupling constant of $0.1 \mathrm{ps}$. The initial configurations were then obtained from the last 450 ps with the time separation of 50 ps. The cell length was adjusted to $19.94 \mathrm{~nm}$ and new velocities were generated.

To address the issue of the influence of ionic arrangement we alternatively placed ions on a grid and kept their positions fixed. The $27 \mathrm{Na}^{+}$ions were placed at ( $i \times a / 3, j \times a / 3, k \times a / 3$ ), where $i, j, k=0,1,2$ and $a$ is the unit cell length. The interionic arrangement of $\mathrm{Cl}^{-}$is the same as that of $\mathrm{Na}^{+}$. The anionic grid is shifted with respect to the cationic one by $1.0 \mathrm{~nm}$ in all three directions as is illustrated in Figure 1. The ionic grid was solvated by 264389 water molecules and the unit cell length was adjusted to $19.94 \mathrm{~nm}$. This was followed by a $600 \mathrm{ps}$ equilibration in NVT ensemble out of which the last 450 ps were used to generate initial configurations for the ten 5 ns production trajectories.

In order to check the influence of Ewald boundary conditions ${ }^{16}$ on the water-water orientational correlation at large distances we performed additional simulations for neat water with two types of Ewald boundary conditions - tin foil $\left(\varepsilon_{\mathrm{r}}{ }^{\prime}=\infty\right)$ and $\varepsilon_{\mathrm{r}}{ }^{\prime}=60$. For 264443 water 
molecules in a $19.94 \mathrm{~nm}$ simulation cell we applied the same computational protocol as for the diluted salt solution and we collected total of $50 \mathrm{~ns}$ of trajectories in total for each of the two boundary conditions.

To examine the system size dependence we simulated pure water in a cell with a half of the previous length. The system contained 33049 water molecules and the production run cell length of $9.972 \mathrm{~nm}$ was determined from an average cell length over the $2 \mathrm{~ns}$ equilibration in the NpT ensemble. The last 200 ps of the equilibration provided 5 initial configurations for subsequent $20 \mathrm{~ns}$ production runs in the NVT ensemble for every configuration. The overall length of the simulation for both types of Ewald boundary conditions was thus $100 \mathrm{~ns}$.

Finally, we also investigated the concentration dependence of the water-water orientational correlation function in a $\sim 10 \mathrm{~nm}$ unit cell. Each of the systems was at first equilibrated for $2 \mathrm{~ns}$ in the $\mathrm{NpT}$ ensemble, the average cell length from this equilibration providing the respective value for the subsequent production run in the NVT ensemble. We collected five $20 \mathrm{~ns}$ trajectories with tin foil boundary conditions for each of the investigated concentration of $17 \mathrm{mM}\left(10 \mathrm{Na}^{+}\right.$and $\left.10 \mathrm{Cl}^{-}\right), 67 \mathrm{mM}\left(40 \mathrm{Na}^{+}\right.$and $\left.40 \mathrm{Cl}^{-}\right), 0.17 \mathrm{M}\left(100 \mathrm{Na}^{+}\right.$and $\left.100 \mathrm{Cl}^{-}\right)$, and $1.0 \mathrm{M}\left(600 \mathrm{Na}^{+}\right.$and $\left.600 \mathrm{Cl}^{-}\right)$.

\section{ACKNOWLEDGMENT}

EP thanks the Czech Science Foundation (grant no. 17-01982Y) and the Czech Academy of Sciences for the Postdoctoral Fellowship (grant no. L200401651). PJ acknowledges support from the Czech Science Foundation (grant no. 16-01074S). The research leading to these results has received funding from the European Research Council under the European Union's Seventh Framework Program (FP7/2007-2013)/ERC Grant Agreement No. 279977 (DL). 


\section{REFERENCES}

1. Evans, D. F. Wennerstrom, H. The Colloidal Domain: Where Physics, Chemistry, Biology, and Technology Meet. Wiley: 1999.

2. Israelachvili, J. Intermolecular and Surface Forces. Academic Press Inc.: 1985.

3. Barthel, J. M. G.; Krienke, H.; Kunz, W. Physical Chemistry of Electrolyte Solutions: Modern Aspects. Springer: 1998.

4. Chen, Y. X.; Okur, H. I.; Gomopoulos, N.; Macias-Romero, C.; Cremer, P. S.; Petersen, P. B.; Tocci, G.; Wilkins, D. M.; Liang, C. W.; Ceriotti, M.; Roke, S. Electrolytes Induce LongRange Orientational Order and Free Energy Changes in the H-bond Network of Bulk Water. Sci. Adv.s 2016, 2, e1501891.

5. Curtis, R. A.; Lue, L. Depletion forces due to image charges near dielectric discontinuities. Current Opinion Colloid Interface Sci. 2015, 20, 19-23.

6. Levrel, L.; Maggs, A. C. Boundary conditions in local electrostatics algorithms. J. Chem. Phys. 2008, 128, 2014103.

7. Kolafa, J.; Viererblova, L. Static Dielectric Constant from Simulations Revisited: Fluctuations or External Field? J. Chem. Theor. Comput. 2014, 10, 1468-1476.

8. Boresch, S.; Steinhauser, O. Presumed versus real artifacts of the Ewald summation technique: The importance of dielectric boundary conditions. Ber. Bunsen-Gesellschaft - Phys. Chem. Chem. Phys 1997, 101, 1019-1029.

9. Vega, C.; Abascal, J. L. F. Simulating water with rigid non-polarizable models: a general perspective. Phys. Chem. Chem. Phys. 2011, 13, 19663-19688.

10. Bagchi, B. Water in Biological and Chemical Processes: From Structure and Dynamics to Function. Cambridge University Press: Cambridge, 2013; p 81-96. 
11. Lynden-Bell, R.; Moris, S. C.; Barrow, J. D.; Finney, J. L.; Harper Jr., C. L. Water and Life: The Unique Properties of H2O. CRC Press: Boca Raton, 2010.

12. Abascal, J. L. F.; Vega, C. A general purpose model for the condensed phases of water: TIP4P/2005. J. Chem. Phys. 2005, 123, 234505.

13. Best, R. B.; Zhu, X.; Shim, J.; Lopes, P. E. M.; Mittal, J.; Feig, M.; MacKerell, A. D. Optimization of the Additive CHARMM All-Atom Protein Force Field Targeting Improved Sampling of the Backbone phi, psi and Side-Chain chi(1) and chi(2) Dihedral Angles. J. Chem. Theor. Comput. 2012, 8, 3257-3273.

14. Kohagen, M.; Mason, P. E.; Jungwirth, P. Accounting for Electronic Polarization Effects in Aqueous Sodium Chloride via Molecular Dynamics Aided by Neutron Scattering. J. Phys. Chem. B 2016, 120, 1454-1460.

15. Hess, B.; Kutzner, C.; van der Spoel, D.; Lindahl, E. GROMACS 4: Algorithms for highly efficient, load-balanced, and scalable molecular simulation. J. Chem. Theor. Comput. 2008, 4, 435-447.

16. Essmann, U.; Perera, L.; Berkowitz, M. L.; Darden, T.; Lee, H.; Pedersen, L. G. A Smooth Particle Mesh Ewald Method. J. Chem. Phys. 1995, 103, 8577-8593.

17. Bussi, G.; Donadio, D.; Parrinello, M. Canonical sampling through velocity rescaling. $J$. Chem. Phys. 2007, 126, 014101.

18. Berendsen, H. J. C.; Postma, J. P. M.; Vangunsteren, W. F.; Dinola, A.; Haak, J. R. Molecular-Dynamics with Coupling to an External Bath. J. Chem. Phys. 1984, 81, 3684-3690. 